\title{
Grundsatzurteil zur medizinischen Begutachtung der Invalidenversicherung
}

\section{Christian Haag}

Rechtsanwalt und Notar Der Autor ist Mitglied des gemeinnützigen Vereins Rechtsberatungsstelle UP für Unfallopfer und Patienten und vertritt regelmässig Versicherte in IV-Streitigkeiten.

* Die Literaturhinweise finden sich unter www.saez.ch

$\rightarrow$ Aktuelle Nummer oder

$\rightarrow$ Archiv $\rightarrow 2011 \rightarrow 51 / 52$

Das Bundesgericht (BGer) hat in einem Grundsatzurteil vom 28.6.2011 [1]* entschieden, dass die Medizinischen Abklärungsstellen (MEDAS) der Invalidenversicherung (IV) sogar dann als unabhängig und neutral gelten, wenn sie finanziell vollständig von der IV abhängig sind. Im IV-Abklärungsverfahren sei zwar die Fairness gefährdet, diese könne aber durch eine Stärkung der Mitwirkungsrechte der Versicherten gewährleistet werden. Das Urteil ändert die Rechtsprechung zum Begutachtungsverfahren und wirft diverse Fragen auf.

\section{Zusammenfassung des Urteils}

Die IV und das kantonale Gericht hatten ein Rentengesuch, gestützt auf ein MEDAS-Gutachten, abgelehnt. Dagegen opponierte die Versicherte vor dem Bundesgericht (BGer).

Das BGer fragte die 18 Schweizer MEDAS zu Auftragsherkunft und Ausmass der attestierten Arbeitsfähigkeit an [2]. Resultat: Der Mittelwert der Anteile von IV-Aufträgen liegt bei 85-90\% [3]. Einige MEDAS erhalten sogar 100\% ihrer Aufträge von der IV [4]. Die drei grossen MEDAS ABI GmbH, MZR Römerhof und Oberaargau beantworteten nicht, in welchem Ausmass sie von 2005 bis 2009 eine (i. d. R. rentenbegründende) Arbeitsunfähigkeit von mind. $40 \%$ für leidensangepasste Tätigkeiten attestierten [5].

\section{Einige Medizinische Abklärungsstellen (MEDAS) erhalten sogar 100 Prozent ihrer Aufträge von der IV.}

Korrespondenz: lic. iur. Christian Haag Häfliger/Haag/Häfliger Rechtsanwälte und Notare Schwanenplatz 7 CH-6000 Luzern 5 ch[at]anwaltluzern.ch
Dies veranlasste das BGer zu folgendem Grundsatzentscheid über das IV-Begutachtungsverfahren: Ein MEDAS-Gutachten hat volle Beweiskraft, auch wenn die IV Auftraggeberin ist [6]. Wirtschaftliche Abhängigkeit einer MEDAS von der IV führt nicht zur Befangenheit [7].

Im Sozialversicherungsverfahren besteht aber ein Ungleichgewicht zugunsten der IV [8]. Die Aufsicht des Begutachtungswesens durch das Bundesamt für Sozialversicherungen (BSV) sei ungenügend, und die aktuelle MEDAS-Situation unbefriedigend [9]. Das Begutachtungsverfahren soll laut BGer mittels nachstehenden Vorschlägen vom BSV fairer ausgestaltet werden [10]:

\section{Arrêt de principe sur l'expertise médicale de l'assurance-invalidité}

Dans son arrêt de principe du 28 juin 2011, le Tribunal fédéral (TF) a décidé que les centres d'observation médicale (COMAI) de l'assurance-invalidité (AI) sont considérés comme indépendants et neutres même s'ils dépendent entièrement de l'Al sur le plan financier. L'impartialité de la procédure d'instruction de l'Al est certes menacée, mais il est possible de la garantir en renforçant les droits de participation des assurés. Cet arrêt modifie la jurisprudence relative à la procédure d'instruction et soulève différentes questions.

- Die MEDAS soll Ergebnisse mit dem behandelnden Arzt diskutieren [11].

- So weit möglich haben sich IV und Versicherter einvernehmlich über eine Gutachterstelle $\mathrm{zu}$ einigen [11].

Zudem beschliesst das BGer folgende Praxisänderungen:

- Wenn sich IV und versicherte Person nicht über die Gutachterstelle einigen können, muss die IV mittels Verfügung festlegen, welche MEDAS mit Hilfe welcher Gutachter welche Fragen beurteilt. Diese Verfügung ist vor Gericht anfechtbar [12].

- Kantonale Gerichte müssen neu vermehrt selber Gerichtsgutachten in Auftrag geben [13].

Bisherige Gutachten werden aber nicht ungültig, sondern sind auf ihre Beweiskraft zu überprüfen [14]. Im konkreten Fall beurteilte das BGer ein MEDAS-Gutachten als nicht schlüssig, weil Differenzen zu einem Privatgutachten nicht überprüfbar und nachvollziehbar begründet wurden. Ein MEDAS-Gutachten muss eine Divergenz zur Einschätzung eines behandelnden Arztes (oder Privatgutachters) umso mehr erörtern, je grösser und wichtiger der Widerspruch ist [15].

\section{Kommentar}

Die Unabhängigkeit der MEDAS trotz finanzieller Abhängigkeit von der IV steht im Widerspruch zur 


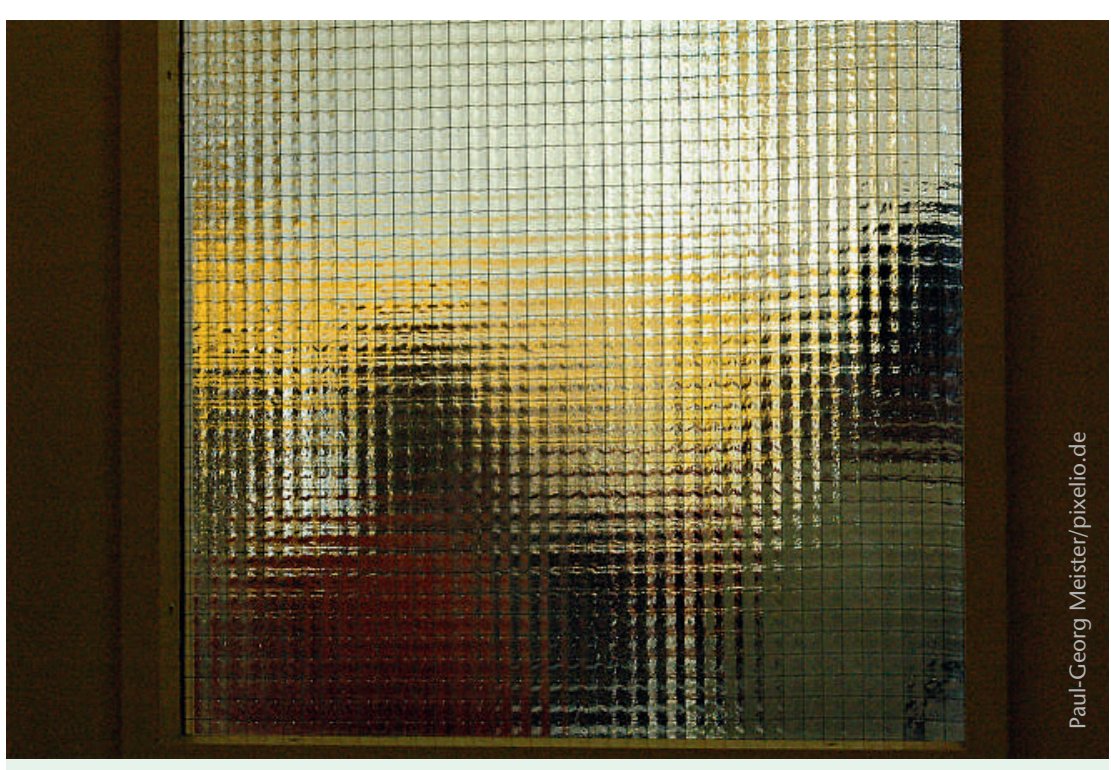

Etwas mehr Transparenz bei der Begutachtungspraxis der Medizinischen Abklärungsstellen der Invalidenversicherung wäre angebracht.

Gerichtspraxis im Straf- und Zivilrecht: Dort haben von einer Verfahrenspartei selber in Auftrag gegebene Gutachten, wenn überhaupt, dann nur eingeschränkte Beweiskraft: Privatgutachter sind nicht wie amtliche Sachverständige unabhängig und unparteiisch, sondern stehen in einem Abhängigkeitsverhältnis zur Auftraggeberin [16]. Dies ist laut BGer bei den MEDAS anders, weil diese von der IV nicht im Ge-

\section{Teilweise wurden MEDAS bevorzugt, die im Sinne des IV-Sparauftrags zulasten der Versicherten begutachten.}

richts-, sondern bereits im Verwaltungsverfahren beauftragt werden, in welchem die IV noch keine Parteistellung habe [17]. Ob diese dogmatische Argumentation verfängt, wird sich anhand des Resultats mehrerer aktuell am Europäischen Gerichtshof für Menschenrechte hängiger Beschwerden zeigen. Jedenfalls hinterlässt der Umstand, dass einzelne MEDAS (gewinnorientierte juristische Personen) 100\% ihrer Aufträge von der IV erhalten und kritische Anfragen des obersten Gerichts nicht beantworten, ein ungutes Gefühl.

\section{Auswirkungen?}

Bis heute konnten IV-Stellen MEDAS nach ihrem Gutdünken auswählen. Teilweise wurden jene MEDAS bevorzugt, die im Sinne des IV-Sparauftrags zulasten der Versicherten begutachten [18]. Aktuell erarbeitet das BSV eine IT-Plattform, um Gutachten nach Zufallsprinzip an Abklärungsstellen zuzuweisen, was laut BGer die Unabhängigkeit fördert [19]. Ein Hauptproblem des aktuellen Begutachtungswesens wird damit aber nicht behoben: Einige der am meisten finanziell abhängigen und am wenigsten transparenten MEDAS zählen zu jenen mit den grössten Kapazitäten für Gutachten [20]. Ihr Marktanteil wird mit einer Gutachtenszuweisung nach Zufallsprinzip nicht kleiner. Es bleibt zu hoffen, dass damit der Druck reduziert wird, im Sinn der IV zu begutachten, um auch künftig Aufträge zu erhalten.

Gerichtlich ungeklärt ist, ob die Rechtsprechung auch für monodisziplinäre und unfallversicherungsrechtliche Gutachten gilt. Nach Ansicht des Schreibenden ist dies zu bejahen, da auch dort eine Abhängigkeitsproblematik besteht und die Verfahrensrechte gefährdet sind [21].

Sodann werden behandelnde Ärzte vermehrt in das Begutachtungsprozedere mit einbezogen:

- Wenn ein Widerspruch bezüglich Diagnose/ Arbeitsfähigkeit besteht, wird eine Rücksprache des Gutachters mit dem behandelnden Arzt vermehrt nötig sein. Art und Umfang dieses Miteinbezugs sind noch nicht festgelegt. Dabei erscheint eine transparente Umsetzung durch schriftliche Anfrage, oder Aktennotiz bei mündlicher Rücksprache, als notwendig. Nur durch Überprüfbarkeit wird das rechtliche Gehör gewährleistet.

- Behandelnde Ärzte müssen damit rechnen, vermehrt von Anwälten mit möglichen Gutachterpersonen und Fragenkatalogen konfrontiert zu werden: Patienten können durch Einwände zu Gutachterpersonen und gezielte medizinische Fragestellung ein Gutachten mitbeeinflussen [11, 12], wozu medizinische Hilfe nötig sein wird.

- Behandelnde Ärzte könnten direkt in Gutachten involviert werden, falls das BSV (wie vom BGer angeregt) den Gutachtern vorschreibt, ihre Ergebnisse mit einem von der versicherten Person bezeichneten Mediziner zu diskutieren [22].

All dies bewirkt eine wünschenswerte und notwendige Stärkung der Stellung behandelnder Ärzte und eröffnet Möglichkeiten, sich für Patienten zu engagieren. Schliesslich ist denkbar, dass vermehrt Gutachten extern vergeben werden: Die Formulierung des BGer schliesst nicht aus, dass für Gerichtsgutachten neben den zu bevorzugenden MEDAS auch andere Gutachter berücksichtigt werden [23].

$\mathrm{Zu}$ guter Letzt empfiehlt das BGer die Schaffung einer Instanz, die fachspezifische, aktuelle Begutachtungsleitlinien erlässt [24].

Viele Fragen zur Tragweite und Umsetzung des BGer-Entscheids sind noch unklar. Am 6.12.2011 findet dazu eine öffentliche Podiumsdiskussion im Kongresshaus Zürich statt. 


\section{Literatur}

1 Fallnummer: 9C_243/2010; wo nicht anders vermerkt, beziehen sich nachfolgende Zitate auf dieses Urteil.

2 Erwägung (E.) 1.2.2 S. 7-11.

3 E. 2.4 .1 S. 48

4 E. 1.2 .3 S. 15 f. und S. 21

5 E.1.2.3 S. 27.

$6 \quad$ E.1.3.4 S. 38.

7 E.1.3.3 S. $37 \mathrm{f}$.

8 E.2.1.2.2 S. 40.

9 E.3.4.2.5 S. 64.

10 E.3.2 S. 54 und E.3.3.2 S. 55.

11 E. 3.1.3.3 S. 54.

12 E.3.4.2.6 \& 3.4.2.7 S. 64 - 66, E.3.4.2.9 S. 67.

13 E.4.4.1.4 S. $72 \mathrm{f}$

14 E. 6 S. 74 .

15 E. 6.2 .4 S. 78
16 LGVE 2001 I Nr. 24; Frank/Sträuli/Messmer, Komm. zur zürcherischen Zivilprozessordnung, 3. Aufl., Zürich 1997, N 4 vor § 171 ff. ZPO/ZH; BGE 132 III 83 E. 4.

17 BGE 136 V 376 E. 4.2.2 S. 380.

18 http://ivinfo.files.wordpress.com/2011/05/ aufsichtsbeschwerde-edi.pdf

19 E. 3.1.2 S. 52 f.

20 E. 1.2.3. S. 15 f., S. 21 f., S. 27: ABI GmbH, MZR Römerhof Zürich, Oberaargau.

21 Gemäss BGer-Urteil 8C_439/2009 ist bereits ein unabhängiges Gutachten nötig, wenn nur schon geringe Zweifel am Gutachten des SUVA-Kreisarztes bestehen.

22 E.3.1.3.3 S. 54.

23 Vgl. Dr. iur. Lendfers Miriam, in: AJP 9/2011, S. 1251, welche z.B. Kantonsspitäler oder Unikliniken erwähnt.

24 E. 3.3.4 S. 56. 